\title{
EFECTO DE LA CASTRACIÓN QUÍMICA EN EL COMPORTAMIENTO PRODUCTIVO Y CONDUCTUAL DEL CUY
}

\author{
Effect of Chemical Castration on the Productive and Behavioral \\ Performance of the Guinea Pig
}

\author{
Jaime Vega V., ${ }^{1,2}$ Hilario Pujada A. ${ }^{1}$, Karina Astocuri C. ${ }^{1}$
}

\section{RESUMEN}

El objetivo del estudio fue determinar el efecto de la castración química con tintura de yodo sobre el comportamiento productivo y conductual del cuy. Se utilizaron 24 cuyes cruzados Perú-Inti, de 25 a 35 días de edad, distribuidos en dos grupos: castrados y no castrados. La castración se realizó en la segunda semana del estudio por inyección intratesticular de tintura de yodo al $2 \%$ en dosis de $0.1 \mathrm{ml}$ por testículo. Se evaluó el peso semanal y al beneficio, ganancia de peso diaria, y el número de peleas, a través de un diseño completamente al azar con covarianza por peso inicial. El peso semanal fue mayor en los castrados a partir de la cuarta semana del estudio (706.5 vs $648.8 \mathrm{~g}, \mathrm{p}<0.05$ ). El peso al beneficio (837.9 vs $738.4 \mathrm{~g}$ ) y la ganancia de peso diaria (9.06 vs $6.94 \mathrm{~g}$ ) fueron mayores en los animales castrados ( $\mathrm{p}<0.01)$. El número de peleas registradas (7.8 vs 14.4$)$ fue menor en los castrados $(\mathrm{p}<0.01)$. Se concluye que la castración química mejora el peso al beneficio y disminuye la conducta agresiva de los cuyes favoreciendo una mejor presentación de la carcasa.

Palabras clave: castración química, cuyes, tintura de yodo, comportamiento, conducta

\section{AbStract}

The objective of the study was to determine the effect of chemical castration using iodine tincture on the productive and behavioral performance of the guinea pig. Twenty four crossbred Peru-Inti guinea pigs from 25 to 35 days of age were used and allocated in two groups: castrated and non-castrated. Iodine tincture at $2 \%$, dose of $0.1 \mathrm{ml}$ per testicle, was used for the castration at the second week of the study. Weekly body weight and at slaughter, daily body weight gain, and number of fights were evaluated using a completely randomized design with covariance for initial body weight. Weekly body weight was higher in castrated animals from the fourth week of the study (706.5 vs 648.8 g) onwards $(\mathrm{p}<0.05)$. Body weight at slaughter (837.9 vs $738.40 \mathrm{~g})$ and daily body weight gain ( $9.06 \mathrm{vs}$ $6.94 \mathrm{~g})$ were higher in the castrated $(\mathrm{p}<0.01)$. The number of fights $(7.8 \mathrm{vs} 14.4)$ was lower

\footnotetext{
${ }^{1}$ Escuela Académico Profesional de Zootecnia (EAPZ), Universidad Nacional José Faustino Sánchez Carrión (UNJFSC), Huacho, Perú

${ }^{2}$ Email: jaimefvv@yahoo.com
} 
in castrated animals $(\mathrm{p}<0.01)$. The chemical castration increases body weight at slaughtering and reduce the aggressive behavior of guinea pigs improving the presentation of the carcasses.

Key words: chemical castration, guinea pigs, iodine tincture, performance, behavior

\section{INTRODUCCIÓN}

La castración en los animales de granja es una práctica ganadera que se establece para facilitar el manejo de los animales (Oteiza y Carmona, 1993). El efecto de la castración varía según la especie, el individuo, la edad y el estado fisiológico en el momento de efectuarla; así por ejemplo, la castración suprime el desarrollo sexual en machos pre-púberes (Hafez, 2002).

La castración química es la más empleada en el caso de cuyes. Al parecer, el primer trabajo publicado sobre castración química se realizó con implantes subcutáneos de dietilestilbestrol (Bravo, 1970) y, posteriormente, a través de inyecciones intratesticulares con tintura de yodo (Hernández, 2001; Rosado, 2001) y alcohol yodado (Shiroma, 2004). En esos trabajos se midió el efecto de la castración sobre la ganancia de peso diaria y el peso al beneficio (Mahan y Lepine, 1991) y la disminución de la agresividad (Pineda, 1991), aunque no se consideraron importantes parámetros como el peso inicial en el primer caso, o el número de peleas en el segundo caso. El presente estudio tuvo por objetivo evaluar el efecto de la castración química con tintura de yodo sobre la ganancia de peso y la agresividad.

\section{Materiales y Métodos}

El estudio se realizó en la Granja Experimental de Zootecnia de la Facultad de Ciencias Agrarias e Industrias Alimentarias, Universidad Nacional José Faustino Sánchez Carrión, Huacho, Lima, entre los meses de abril y mayo de 2010.
Se utilizaron 24 cuyes cruzados PerúInti, de 25 a 35 días de edad. Se formaron dos grupos de doce animales asignados al azar: un grupo control (no castrados) y un grupo castrado. La castración se realizó mediante inyección intratesticular de tintura de yodo al $2 \%$ en dosis de $0.1 \mathrm{ml}$ por testículo. El peso inicial del grupo castrado fue de 368.8 $\pm 20.3 \mathrm{~g}$ y el del grupo no castrado fue de $425.8 \pm 14.2$ g (promedio \pm error estándar). La castración se realizó a las dos semanas después de iniciado el experimento.

Cada grupo se alojó en jaulas de $90 \mathrm{x}$ $90 \mathrm{~cm}$, con bebederos y comederos de arcilla. Se les proporcionó alimento balanceado ad libitum, el cual contenía $2953 \mathrm{kcal} / \mathrm{kg}$ de energía digestible (ED) y 19.08 \% de proteína total (PT), empleando los insumos señalados en el Cuadro 1. El suministro de vitamina C fue a través de forraje verde (maíz chala) en la primera semana de iniciado el estudio y posteriormente a través del agua de bebida.

Cuadro 1. Insumos utilizados en la ración

\begin{tabular}{ll}
\hline Insumo & Porcentaje \\
\hline Maíz grano & 32.24 \\
Afrecho de trigo & 43.27 \\
Torta de soya & 16.34 \\
Pasta de algodón & 3.69 \\
Harina integral de soya & 2.76 \\
Carbonato de cal cio & 1.09 \\
Sal & 0.36 \\
Premezcla & 0.25 \\
\hline Total & 100.00 \\
\hline
\end{tabular}


Cuadro 2. Comportamiento productivo de los cuyes castrados y no castrados (promedio \pm error estándar)

\begin{tabular}{lccc}
\hline Grupo & Peso al beneficio $(\mathrm{g})$ & $\begin{array}{c}\text { Ganancia de peso } \\
\text { diaria }(\mathrm{g})\end{array}$ & Peleas $\left(\mathrm{N} .{ }^{\circ}\right)$ \\
\hline Castrado & $837.9 \pm 18.7^{\mathrm{a}}$ & $9.06 \pm 0.38^{\mathrm{a}}$ & $7.80 \pm 0.74^{\mathrm{a}}$ \\
No castrado & $738.4 \pm 18.7^{\mathrm{b}}$ & $6.94 \pm 0.38^{\mathrm{b}}$ & $14.40 \pm 0.74^{\mathrm{b}}$ \\
\hline
\end{tabular}

${ }^{a, b}$ Superíndic es distintos dentro de grupos indican diferencia estadística $(\mathrm{p}<0.01)$

El peso fue controlado semanalmente por un periodo de siete semanas, utilizando una balanza con aproximación de 5 g. El número de peleas dentro de cada grupo fue registrado en los últimos diez días del estudio, mediante observación visual dos horas después del suministro del alimento matutino y por espacio de 30 minutos.

Las variables evaluadas fueron peso semanal, peso al beneficio, ganancia de peso diaria y número de peleas. Se empleó un modelo completamente al azar con covarianza (Kuehl, 2001), considerando la variable peso al inicio del experimento para ajustar los pesos semanales y el peso al beneficio (Mahan y Lepine, 1991). Se empleó el programa MINITAB para el análisis estadístico.

\section{Resultados y Discusión}

El promedio de peso al beneficio y ganancia diaria de peso, ajustados por peso inicial, se muestran en la Cuadro 2. El peso al beneficio de los animales castrados fue 99.5 g superior al grupo no castrado $(\mathrm{p}<0.01) \mathrm{y}$ de $2.12 \mathrm{~g}$ en la ganancia diaria de peso $(p<0.01)$. Estos resultados se explican por el menor gasto de energía en el metabolismo basal en los animales castrados debido a la ausencia de las hormonas sexuales (Bondi, 1988).

Los resultados del presente estudio fueron relativamente menores a lo señalado por Hernández (2001), Rosado (2001) y Shiroma
(2004), posiblemente debido al empleo de cuyes cruzados y por el menor periodo de engorde (7 semanas), dado que en los otros estudios se utilizaron cuyes de raza Perú por 8 semanas. Por otro lado, en esos estudios no se pudo determinar el efecto positivo de la castración al no haberse hecho la corrección de las variables evaluadas por el peso inicial. En el presente estudio, al utilizar la covarianza por peso inicial, el efecto real de la castración pudo ser determinado.

La evolución del peso vivo semanal, ajustado por la covariable peso inicial se muestra en la Fig. 1. La castración se realizó al final de la segunda semana y ambos grupos tuvieron pesos similares hasta la tercera semana de iniciado el estudio. El efecto de la castración sobre el peso es mayor para los animales castrados en la cuarta semana (706.5 vs $648.8 \mathrm{~g}, \mathrm{p}<0.05)$ y con diferencias altamente significativas a partir de la quinta semana $(\mathrm{p}<0.01)$.

El número de peleas en el grupo de animales castrados fue la mitad en relación a los animales testigo (Cuadro 1, $\mathrm{p}<0.01$ ), corroborando lo mencionado por Pineda (1991), quien indica que la castración disminuye la agresividad. Criaderos con altas densidades poblacionales pueden tener problemas de manejo propios de la sobrepoblación (Celis y Saravia, 1999), y es en estos casos donde la castración podría disminuir el número de peleas y permitir una mejor respuesta productiva. En el presente estudio se utilizó una den- 


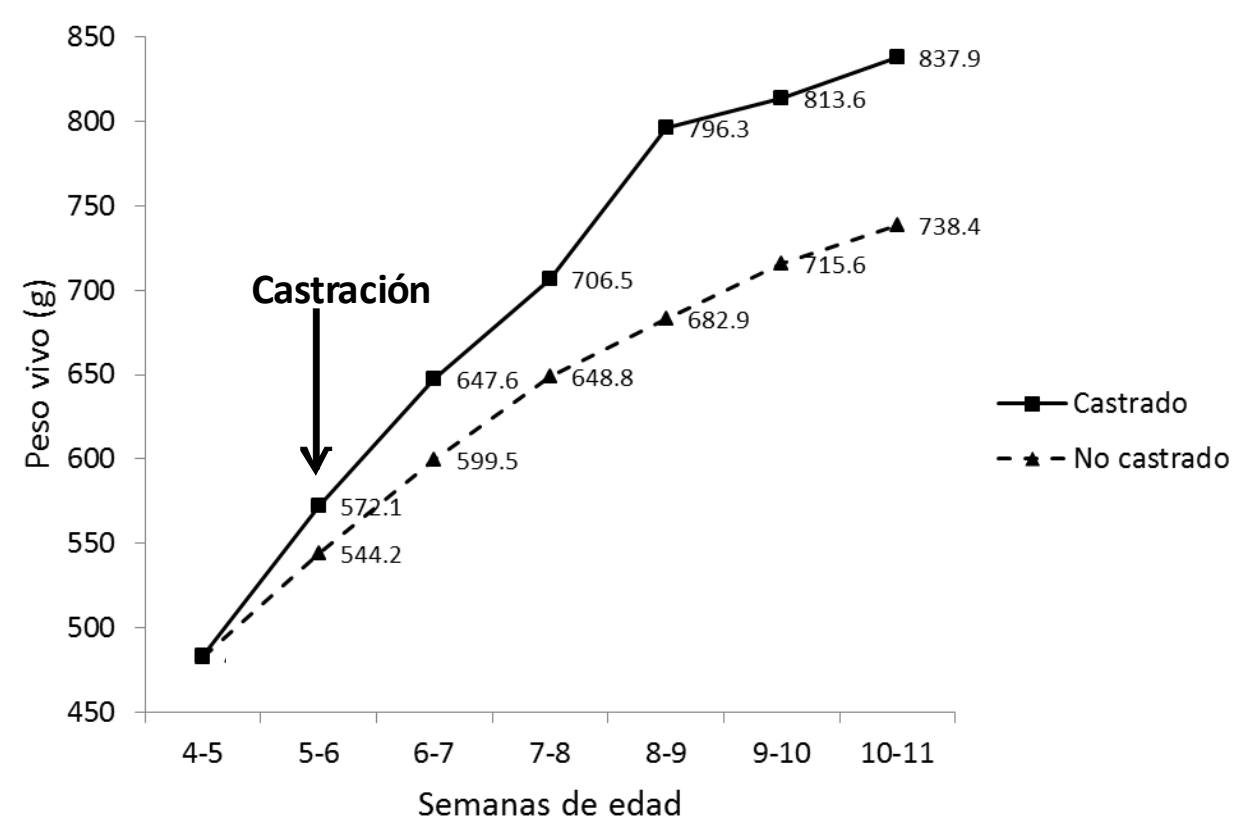

Figura 1. Evolución del peso semanal ajustado en cuyes sin castrar y castrados a la segunda semana del estudio (39 a 49 días de edad)

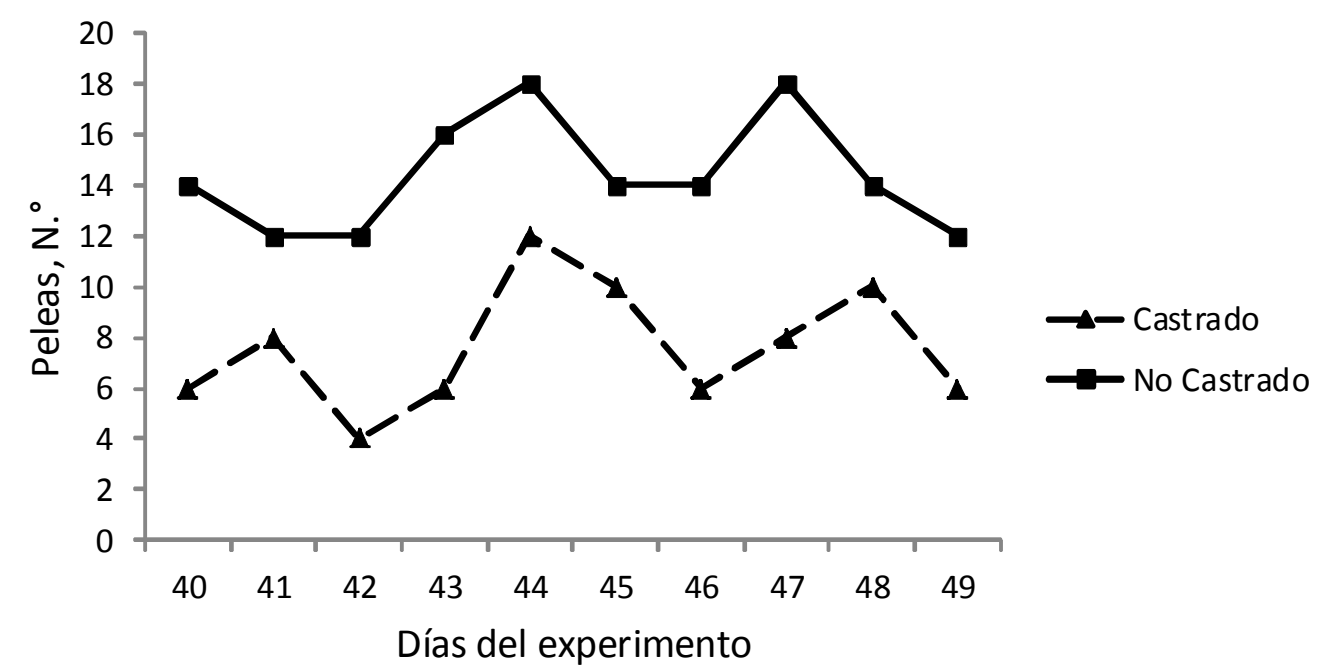

Figura 2. Patrón de comportamiento agonístico en cuyes sin castrar y castrados a la segunda semana del estudio 


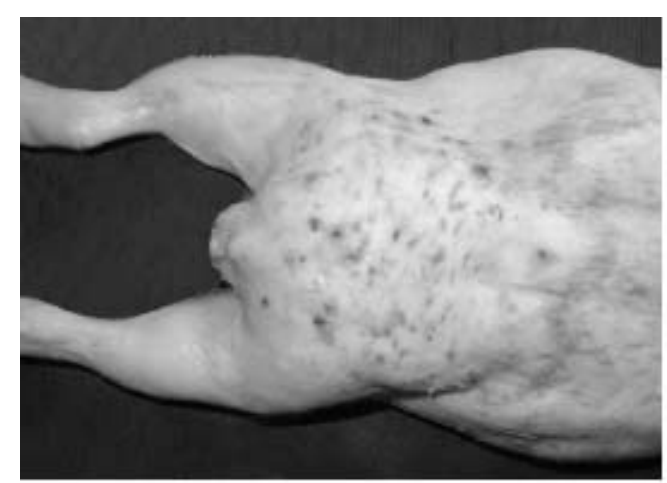

(a)

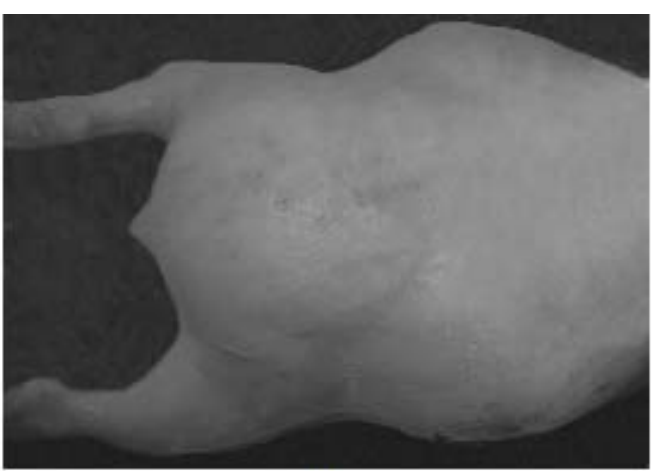

(b)

Figura 3. Carcasas de cuy. (a) Sin castrar mostrando laceraciones evidentes en el lomo por efecto del comportamiento agonístico. (b) Cuy castrado sin que se pueda observar lesiones.

sidad de $0.07 \mathrm{~m} 2$ /animal, donde se observó un patrón de comportamiento similar en ambos grupos, siendo la diferencia el número de peleas (Fig. 2). Por otro lado, las carcasas de los animales castrados no presentaron signos evidentes de laceraciones, en tanto que las carcasas de los cuyes sin castrar presentaron muchas laceraciones (Fig. 3).

\section{Conclusión}

La castración química mejora el peso al beneficio y disminuye la conducta agresiva de los cuyes favoreciendo una mejor presentación de la carcasa.

\section{Agradecimientos}

Los autores agradecen a la Dirección de la EAPZ-UNJFSC por el financiamiento y las facilidades brindadas para realizar la presente investigación.

\section{Literatura Citada}

1. Bondi AA. 1988.Nutrición animal. España: Acribia. 546 p.
2. Bravo H. 1970. La implantación de dietilestilbestrol en cuyes y sus efectos. Tesis de Ingeniero Zootecnista. Lima: Universidad Nacional Agraria La Molina. $60 \mathrm{p}$.

3. Celis E, Saravia J. 1999. Detección de dermatomicosis en cuyes criados en batería y pozas. En: XXII Reunión APPA. Huancavelica: Asociación Peruana de Producción Animal.

4. Hafez ESE. 2002. Anatomía del aparato reproductor del macho. En: Hafez ESE, Hafez B (eds). Reproducción e inseminación artificial en animales. 7a ed. México: McGraw-Hill. p 3-12.

5. Hernández ME. 2001. Comparación entre castración química y quirúrgica de cuyes en la etapa de engorde. Tesis de Médico Veterinario y Zootecnista. Arequipa: Universidad Católica de Santa María. 46 p.

6. Kuehl RO. 2001. Diseño de experimentos. $2^{\text {a }}$ ed. México: Thompson Learning. $666 \mathrm{p}$.

7. Mahan DC, Lepine AJ. 1991. Effect of pig weaning weight and associated nursery feeding programs on subsequent performance to 105 kilograms body weight. J Anim Sci 69: 1370-1378. 
8. Oteiza J, Carmona JR. 1993. Diccionario de zootecnia. $3^{\text {a }}$ ed. México: Trillas. $316 \mathrm{p}$.

9. Pineda MH. 1991. Reproducción del macho. En: McDonald LE, Pineda MH (eds). Endocrinología veterinaria y reproducción. $4^{\mathrm{a}}$ ed. México: McGraw-Hill.p 253-293.

10. Rosado RE. 2001. Estudio comparativo de peso entre cobayos castrados me diante punción y castración quirúrgi- camente en el distrito de Cerro Colorado. Tesis de Médico Veterinario y Zootecnista. Arequipa: Universidad Católica de Santa María. 62 p.

11. Shiroma P. 2004. Efecto de la castración química con alcohol yodado sobre el crecimiento y rendimiento de la canal en cuyes (Cavia porcellus). Tesis de Médico Veterinario. Lima: Universidad Alas Peruanas. $41 \mathrm{p}$. 